\title{
Proximal humerus chondrosarcoma in a young male patient
}

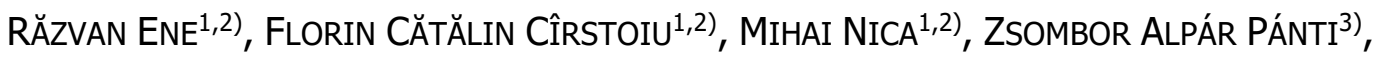

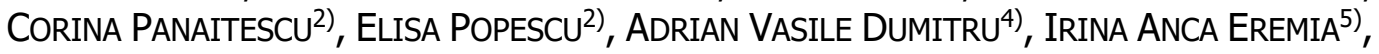 \\ ADRIAN CURSARU ${ }^{1,2)}$, DRAGOŞ ENE ${ }^{6)}$
}

\author{
1) Department of Orthopedics and Traumatology, Carol Davila University of Medicine and Pharmacy, \\ Bucharest, Romania \\ 2) Department of Orthopedics and Traumatology, University Emergency Hospital of Bucharest, \\ Romania \\ 3) Department of Orthopedics, Semmelweis University, Budapest, Hungary \\ 4) Department of Pathology, University Emergency Hospital of Bucharest, Romania \\ 5) Department of Emergency, University Emergency Hospital of Bucharest, Romania \\ 6) Department of Surgery, University Emergency Hospital of Bucharest, Romania
}

\begin{abstract}
Proximal humerus chondrosarcoma is a rare localization of the common primary malignant cartilaginous tumor. Management is based on oncological surgical excision because of the inherent resistance to chemotherapy and radiation therapy. The most important prognostic factors with great impact on treatment strategy are histological grading and localization of the tumor. Proximal humerus chondrosarcoma carries a slightly better prognosis, especially for the low and moderate grade tumors. We present the case of a young patient with proximal humerus chondrosarcoma surgically managed in our Department, with the purpose of underlining the challenges posed by this localization for oncological excision and reconstruction but also the importance of histological grading for the treatment algorithm.
\end{abstract}

Keywords: chondrosarcoma, proximal humerus, excision, reconstruction.

\section{口 Introduction}

Chondrosarcoma is a malignant cartilaginous tumor identified by its characteristic histological composition based on hyaline cartilage. It is the third most common primary malignant bone tumor overall, and the second most common primary malignant bone neoplasm of adults, after myeloma $[1,2]$. Most of the chondrosarcoma cases are sporadic but they can also occur because of malignant transformation of benign tumors like enchondromas or osteochondromas. This malignant transformation ensues on solitary, benign lesions or, with a much higher incidence, on one of the multiple lesions found in patients with Maffucci syndrome or Ollier's disease; the axial skeleton is frequently affected (sternum, pelvis, scapula, or ribs) followed by the proximal femur and humerus [3]. The epidemiological characteristics of chondrosarcoma are the slight male predominance and the highest prevalence in patients older than 50 years of age. Pathogenesis of chondrosarcoma is not clearly understood with higher and higher focus on the importance of genetic factors like chromosomal structural abnormalities and transcription factors in the development of this pathology. Histopathology classifies chondrosarcoma into a conventional type (80$90 \%$ of cases) and non-conventional forms like mesenchymal chondrosarcoma, dedifferentiated chondrosarcoma, or clear cell chondrosarcoma. The standard microscopic examination reveals prolific extracellular cartilaginous matrix and atypical, heterogeneous chondrocytes with large hyperchromatic nuclei. Areas of myxoid change, calcification or necrosis are common findings for chondrosarcoma $[4,5]$.

A particularly important feature is the histological grading system of chondrosarcoma, which has essential prognostic significance and guides the treatment strategy. Grade I tumors are characterized by a histological appearance resembling enchondroma, with uniform, hyperchromatic nuclei, and moderate cellularity. These are considered lowgrade lesions. Grade II tumors have a higher level of nuclear atypia and a more aggressive behavior. Grade III lesions possess greater nuclear and cellular pleomorphism, with frequent mitoses and poorer differentiation. The worst prognosis is carried by dedifferentiated chondrosarcoma, which is a high-grade neoplasm, with spindled cells, high nuclear atypia, and little cartilaginous matrix $[6,7]$.

Clinical presentation is marked by nonspecific symptoms like swelling and localized pain, which manifest for long periods of time until diagnosis is established. Plain radiography is the preferred imaging technique for initial diagnosis. It usually reveals lytic bone lesions with a high incidence of intralesional calcifications (the socalled "popcorn pattern") but also permeating lesions, specific for the aggressive forms which can lead to pathological fracture $[8,9]$. A comprehensive evaluation of the primary tumor and possible secondary bone lesions is accomplished using computed tomography (CT), which can reveal heterogeneous intensification, cortical disruption and matrix calcification complemented with magnetic resonance imaging (MRI) and scintigraphy.

This is an open-access article distributed under the terms of a Creative Commons Attribution-NonCommercial-ShareAlike 4.0 International Public License, which permits unrestricted use, adaptation, distribution and reproduction in any medium, non-commercially, provided the new creations are licensed under identical terms as the original work and the original work is properly cited. 
The management is governed by surgery with wide excision for intermediate and high-grade lesions, axial skeleton tumors, with soft tissue or joint involvement $[10,11]$. Central, low-grade tumors can be excised by curettage and adjuvant, local therapy [phenol, hydrogen peroxide, liquid nitrogen, poly(methyl methacrylate) (PMMA)] [12, 13]. Chemotherapy has limited use and only for high-grade tumors like the dedifferentiated type [14]. Radiation therapy can be used for unresectable lesions or as adjuvant therapy after surgery for local control of recurrence [15]. The most important prognostic factor for chondrosarcoma is the histological grade. The five-year survival rate ranges from $83 \%$ for patients with grade I lesions to less than $60 \%$ for grade II or III chondrosarcoma, and to even lower numbers for the dedifferentiated type $[16,17]$.

\section{Aim}

We present the case of a 46-year-old male, diagnosed and treated in our Department for a grade II chondrosarcoma of the proximal humerus with the scope of underlining the particularities of this disease localization and the reconstruction challenges generated by it and also to emphasize the importance of oncological excision in order to ensure a favorable, long-term result.

\section{Case presentation}

We report the case of a 46-year-old male who was admitted in the Department of Orthopedics and Traumatology, University Emergency Hospital of Bucharest, Romania, for pain and limited range of motion in his right shoulder. No history of trauma or other significant medical history was revealed, with a minimum of six months of nagging pain described by the patient. He managed to control the pain for a short period of time by taking non-steroidal anti-inflammatory drugs (NSAIDs) before the pain became impervious to medication. No family history of malignancy was disclosed by the patient. Routine laboratory tests did not reveal any important changes except for a mild increase in the erythrocyte sedimentation rate (ESR).

Physical examination documented decreased range of motion in the right shoulder joint with mild limitation of abduction, painful passive, and a hard, fixed mass of the proximal humerus on palpation. No axillary adenopathy was accounted for and no neurological symptoms or pulse deficit distally. A standard antero-posterior X-ray of the shoulder showed a lytic lesion of the proximal humerus, with endosteal scalloping and thinned cortices (Figure 1). For tumoral extension assessment, we ordered a CT scan of the involved proximal limb, thorax, abdomen, and pelvis and a bone scintigraphy.

CT scan revealed a lytic lesion, with a craniocaudal dimension of $87 \mathrm{~mm}$ and 81 by $70 \mathrm{~mm}$ in the transversal plane, areas of cortical thinning or cortical breach and periosteal reaction (Figure 2).

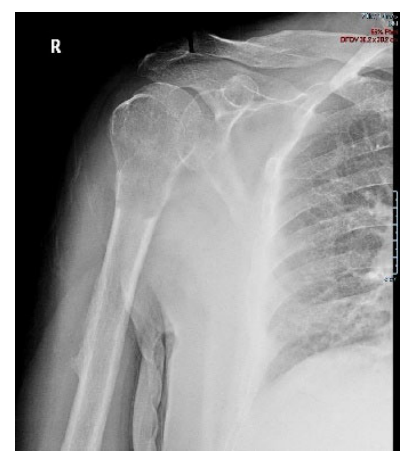

Figure 1 - Proximal humerus lytic lesion with metaphyseal and epiphyseal involvement.
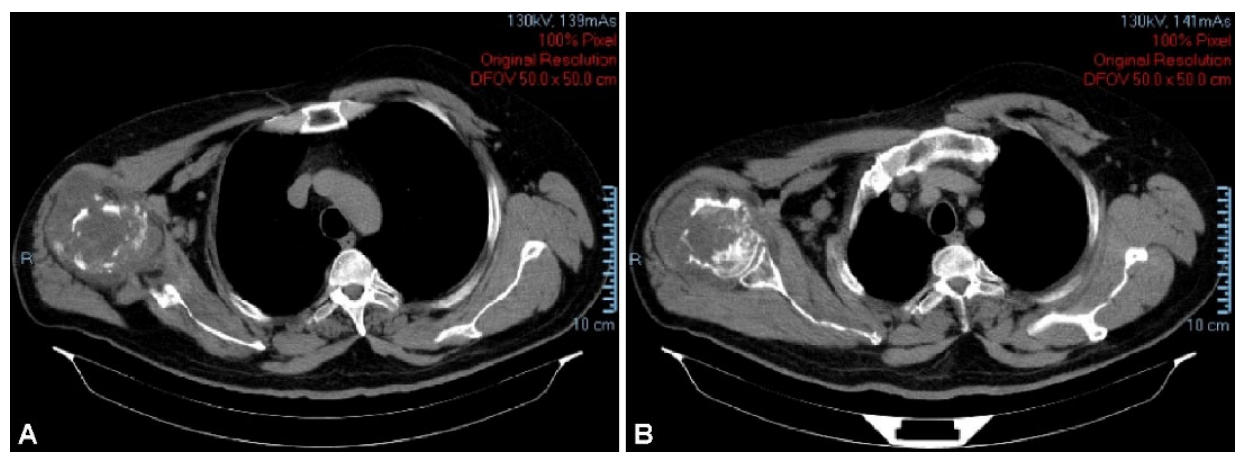

Figure 2 - (A and B) CT scan showing proximal diaphyseal and humeral head involvement with extensive bone lysis and calcification areas. CT: Computed tomography.
The tumor exerted compression on the adjacent infraspinatus muscle tendon, long head of biceps and anterior deltoid. Furthermore, minimally invasion of the articular space but no axillary, brachial arterial branches or brachial plexus involvement were detected. A bone scan using Technetium-99m-Hydroxymethylene diphosphonate ( ${ }^{99 m}$ Tc-HDP) with a dosage of $629 \mathrm{MBq} / 5032 \mathrm{mSv}$ was performed, which revealed intense fixation in the proximal humerus, involving the proximal diaphysis, metaphysis, and humeral head (Figure 3).

The comprehensive imagistic and clinical evaluation did not reveal any secondary lesions. An incisional biopsy centered on the tumor was performed and the tissue sample was sent for histopathology and immunohistochemistry analysis. The reports showed a diagnosis of grade II conventional chondrosarcoma with positive S100 protein inside the cells and actin in the blood vessels (Figures 4-6). Also, the Ki67 proliferation immunomarker was positive with an index of $10 \%$.
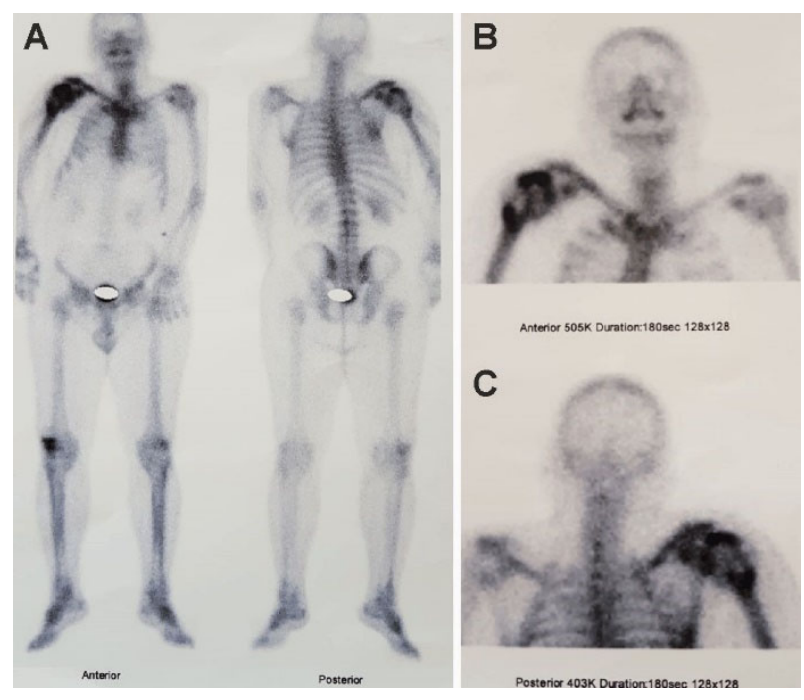

Figure $3-(A-C)$ Technetium-99m bone scan showing intense fixation in the right proximal humerus. 


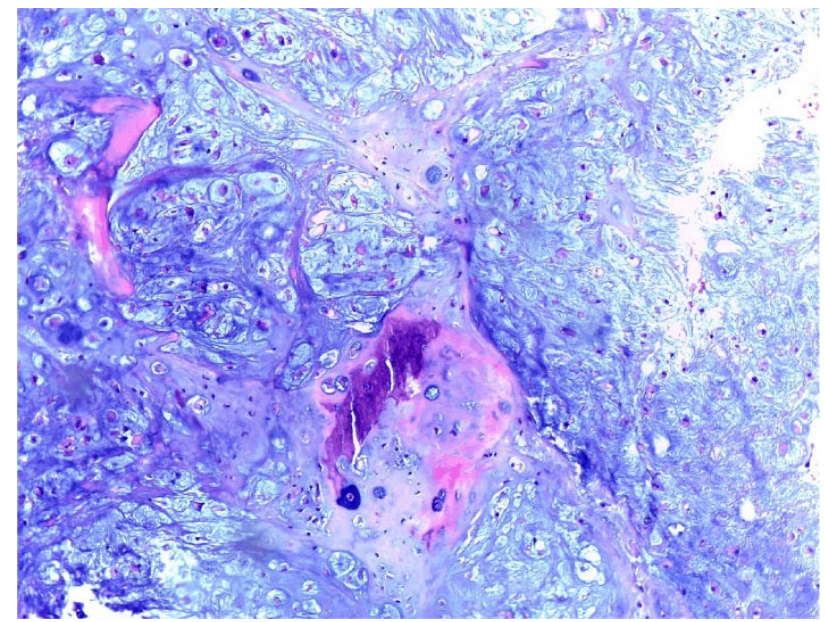

Figure 4 - Moderate-grade (grade 2) conventional chondrosarcoma demonstrating increased cellularity, binucleation and multinucleation, and an increased nuclear-to-cytoplasmic ratio. A lobular arrangement is noted with stroma between the lacunar spaces and few non-neoplastic dystrophic calcifications. HE staining, $\times 100$. HE: Hematoxylin-Eosin.

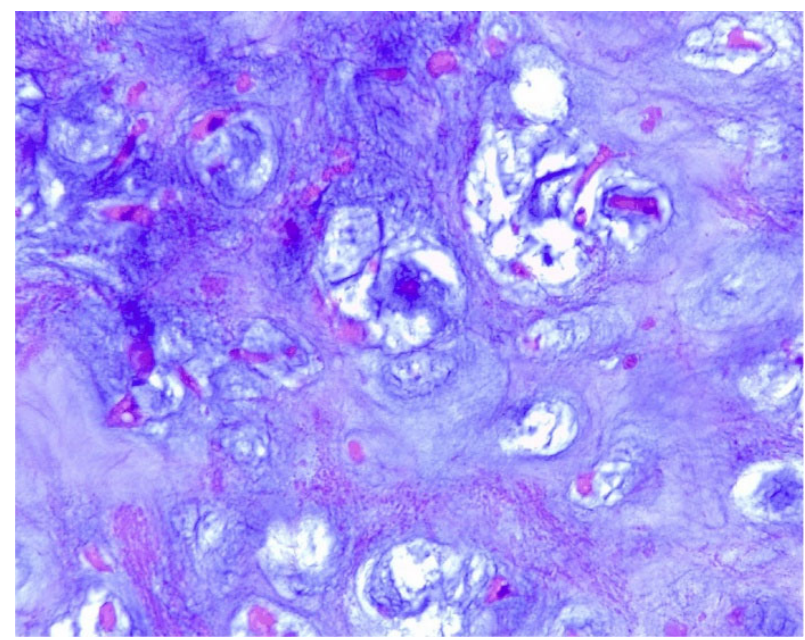

Figure 6 - High power magnification showing atypical tumor cells. Most nuclei are larger, irregular. Note the intracytoplasmic hyaline globule (commonly found in well and moderately differentiated chondrosarcoma). HE staining, $\times 400$.

A clear-cut indication for a limb salvage procedure with surgical removal of the tumor and reconstruction was established, to which the patient was initially resistant, against medical advice, delaying the surgery by four months. During the delay, the patient reported that he attempted different homeopathic therapies with questionable effects on the disease progression. At the time of surgery, we could clearly establish a progression of the bone lysis on plain X-rays, which prompts the question on the influence of the surgery delay combined with the alternative therapies experimented by the patient (Figure 7). The CT scans were repeated before surgery for a comprehensive evaluation of the disease extension.

According to established treatment protocol, a wide excision of the tumor was planned for and executed using an extended deltopectoral approach, with the patient in beach-chair position, under general anesthesia. By careful dissection, removal of the proximal humerus was

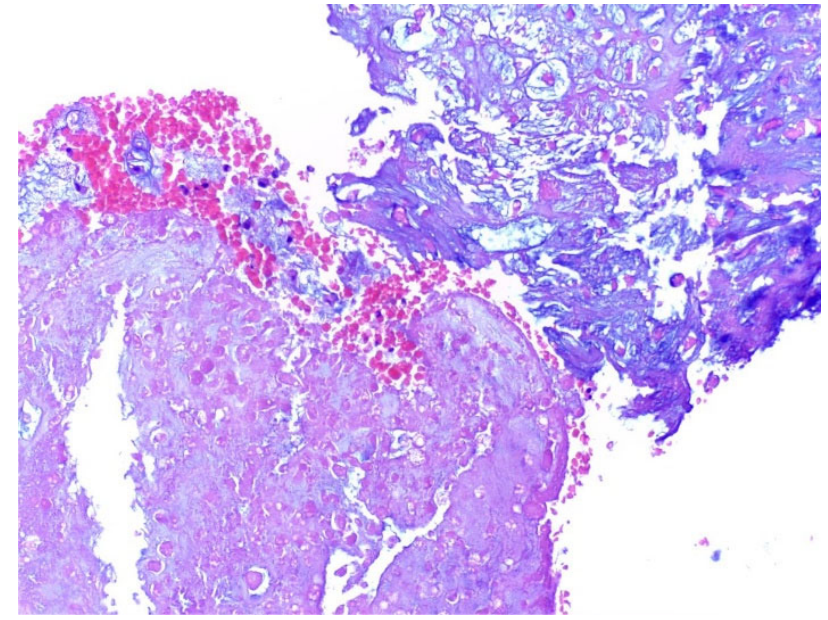

Figure 5 - Tumor necrosis and hemorrhagic areas in a grade 2 chondrosarcoma. HE staining, $\times 100$.

performed with the distal cut-off point at $8 \mathrm{~cm}$ below the surgical neck, which unfortunately forced us to disrupt a limited part of the deltoid insertion on the humeral diaphysis. Meticulous dissection was executed, making sure that the resection limits are negative at the histopathology examination. The reconstruction method employed was a partial humeral arthroplasty using Neer-type prosthesis with a monobloc, cemented humeral stem (Figure 8). The acknowledged difficulty of proximal humeral reconstruction in restoring the rotator cuff function was tackled by careful approximation using nonabsorbable sutures of the remaining subscapularis muscle to the deltoid muscle and also fixation of the external rotator muscles to the implant metaphysis, in the specially designed area, which acts as a greater humeral tuberosity (Figure 9). Further soft tissue reconstruction and hemostasis were performed followed by layered wound closure with closed suction drainage.

The immediate postoperative course of the patient was uneventful, with hospital discharge at seven days after surgery. Rehabilitation was started during the hospital stay by passive joint mobilization and the upper limb was put in a shoulder sling immobilizer as part of the postoperative protocol after all surgical procedures that aim at joint stability restoration trough soft tissue healing. This can help avoid any kind of mechanical complications of the reconstruction while allowing a fair progress of the rehabilitation protocol [18-20]. No radiation therapy or chemotherapy were implemented after surgery. Histopathology results of the resected tissue showed the same grading compared to initial diagnosis, with all resection margins negative for tumoral involvement. The functional results were poor, with the patient failing to recover much of the shoulder active movement but with fair joint stability and good pain control. The rotational mobility was greatly affected, and abduction limited to only $30^{\circ}$. The oncological results are sustainable with one-year disease-free at this moment and no other type of complications, according to Henderson classification [21]. 


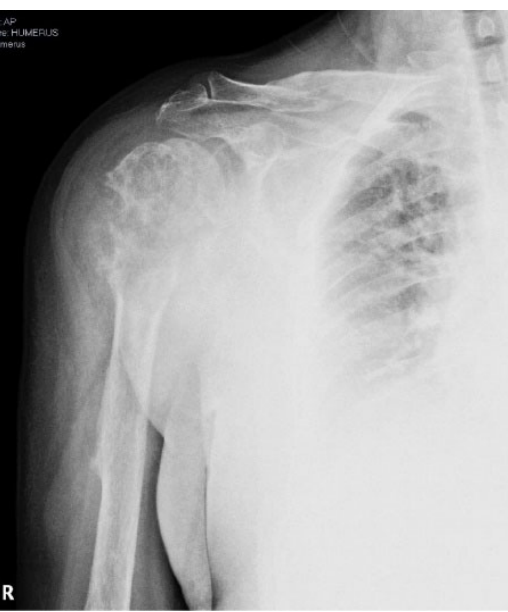

Figure 7 - Preoperative X-ray at four months after biopsy showing progression of the bone lytic lesion.

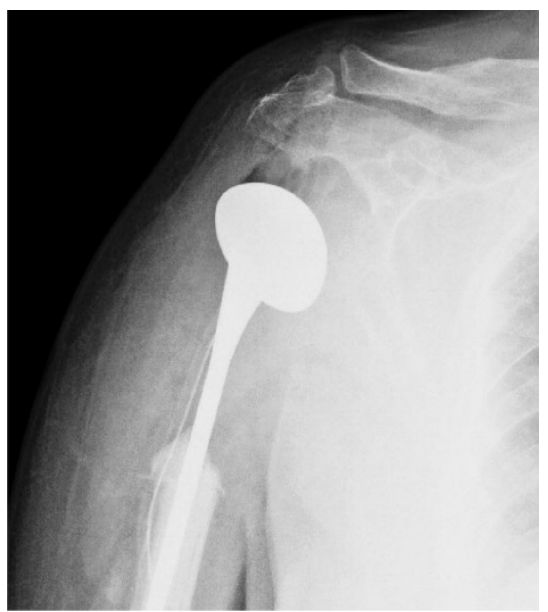

Figure 8 - Postoperative X-ray showing proximal humerus reconstruction with Neer-type prosthesis.

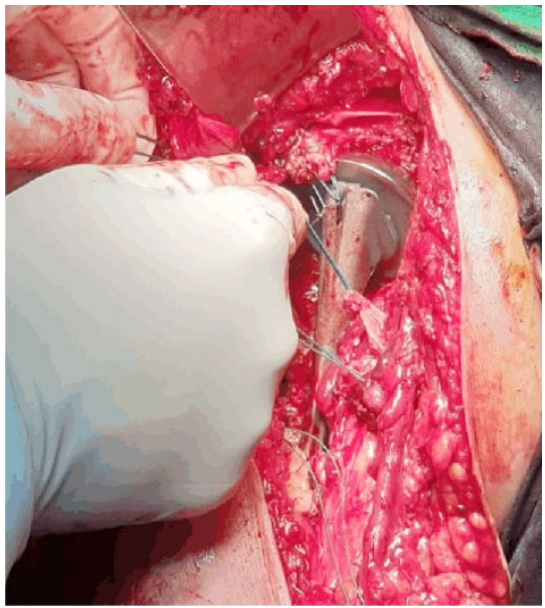

Figure 9 - Intraoperative image showing the approximation of the external rotators and long head of biceps at the implant metaphyseal area.

\section{ㅁ Discussions}

Proximal humerus localization accounts for a small percentage out of the total number of chondrosarcoma cases, and there is a small number of papers about this tumor group [22]. As chondrosarcoma presents with resistance to chemotherapy and radiation therapy achieved through multiple genetic and molecular mechanisms, surgery remains the pillar of treatment [23-25]. The advancements in medical imaging, implant design and surgical techniques have allowed limb sparing procedures for most patients, avoiding the mutilating scapulohumeral disarticulation, which was the favored treatment option in the past. Brachial plexus or axillary vascular structures involvement remain the leading findings that can motivate a disarticulation procedure nowadays [26-28].

Although chondrosarcoma of the proximal humerus has a better oncological prognosis than other localizations of the same disease, histological grading remains the main predictive factor for outcome. There is a great variety of reconstruction techniques which can be employed after tumor resection of the proximal humerus, using: osteoarticular allograft, free or vascularized peroneal autograft, endoprosthesis or allograft-prosthesis composite (APC). Presently, there is no consensus on which reconstruction method yields the best results, with the reverse shoulder arthroplasty being implied as an advisable technique for obtaining superior functional results [29-31]. The main objectives after proximal humeral reconstruction, in terms of good functionality are recovery of joint mobility (active and passive) and stability. The complex anatomy of the shoulder region coupled with all these factors assigns a high level of difficulty for the proximal humerus reconstruction surgery.

The management strategy of our case was based on these principles and adapted to the particularities of the situation. We had the concern that the delay in treatment caused by the patient and the unknown effects of alternative therapies administered during that time could have increased the histological grading of the tumor. This consideration was based mainly on changed imagistic appearance at the time of surgery compared to the moment of biopsy, which suggested a fast, local progression of the tumor. The observed changes were a slight increase in size, bone destruction and soft tissue involvement but also an escalation of pain, causing severe restriction of movement. All these factors coupled with histological grading and the joint involvement, observed on the CT scan suggested that the necessary wide resection will probably require a great soft tissue sacrifice and direct us from a Malawer type I, intra-articular proximal humeral resection to a type $\mathrm{V}$ extra-articular proximal humeral and glenoid resection with devastating functional effects [32]. After thorough consultation with the patient and with the multidisciplinary team (pathologist, oncologist, plastic surgeon, vascular surgeon, and rehabilitation specialist), we decided that we will choose the IA resection type if no gross, macroscopic invasion of the joint space is detected during surgery.

The surgical excision was performed according to this strategy with preservation of the glenoid and most of the deltoid insertion. Because of the need for oncological resection, the insertions of the rotator cuff, latissimus dorsi, teres major, pectoralis major, long head of the biceps and the axillary nerve had to be sacrificed, making a wrapping reconstruction of the rotator cuff impossible [33]. The histopathology examination reported the same grading for the excised tumor (grade II chondrosarcoma) and the resection margins were declared negative. A predictable level of joint dysfunction resulted after surgery with important limitation of active abduction to a maximum of $30^{\circ}$ and almost impossible active rotational mobility. Pain control and joint stability was maintained through all the postoperative period along with good oncological result. Despite the impaired functional result, we expect that the favorable oncological outcome and humeral reconstruction will endure as predicted by the histological grade and localization of the treated chondrosarcoma [34]. No disease recurrence or other complications emerged until present time and continuous follow up of the patient is being undertaken. 
According to our experience, the challenging management of proximal humerus chondrosarcoma stands out, stemming from the delicate balance that have to be maintained between the extensive resection dictated by oncological principles and the need for preservation of the intricate shoulder anatomy and complex reconstruction procedures in order to achieve limb preservation and, if possible, a good functional result. The possibility of dissimilar histopathological grade between the final resection piece and biopsy sample analysis makes the treatment even more complicated sometimes. This prompts for development of new treatment strategies to improve the management of chondrosarcoma, in general, and for proximal humerus localization. Promising research is currently directed towards the elucidation of the mechanisms for chemotherapy and radiation therapy resistance and development of targeted therapies with synergistic effect with the conventional options [35].

\section{a Conclusions}

The contemporary management of chondrosarcoma in founded on oncological surgical excision due to its resistance to radiation and chemotherapy. Proximal humerus chondrosarcomas have a slightly better prognosis compared to other disease localizations but with added challenges for surgical management. The actual treatment strategy involves limb saving procedures, with complex reconstruction techniques employed after oncological resection, which carries the risk for specific complications. Improvement of reconstruction techniques and overall chondrosarcoma management is required, with the final goal of better oncological and functional results.

\section{Conflict of interests}

The authors declare that they have no conflict of interests.

\section{References}

[1] Rozeman LB, Cleton-Jansen AM, Hogendoorn PCW. Pathology of primary malignant bone and cartilage tumours. Int Orthop 2006, 30(6):437-444. https://doi.org/10.1007/s00264-006-02 12-x PMID: 16944143 PMCID: PMC3172744

[2] van Praag Veroniek VM, Rueten-Budde AJ, Ho V, Dijkstra PDS Study Group Bone and Soft Tissue Tumours (WeBot); Fiocco M, van de Sande MAJ. Incidence, outcomes and prognostic factors during 25 years of treatment of chondrosarcomas. Surg Oncol, 2018, 27(3):402-408. https://doi.org/10.1016/j.suronc.2018. 05.009 PMID: 30217294

[3] Verdegaal SHM, Bovée JVM, Pansuriya TC, Grimer RJ, Ozger H, Jutte PC, San Julian M, Biau DJ, van der Geest ICM, Leithner A, Streitbürger A, Klenke FM, Gouin FG, Campanacci DA, Marec-Berard P, Hogendoorn PCW, Brand R, Taminiau AHM. Incidence, predictive factors, and prognosis of chondrosarcoma in patients with Ollier disease and Maffucci syndrome: an international multicenter study of 161 patients. Oncologist 2011, 16(12):1771-1779. https://doi.org/10.1634/theoncologist. 2011-0200 PMID: 22147000 PMCID: PMC3248776

[4] Qasem SA, DeYoung BR. Cartilage-forming tumors. Semin Diagn Pathol, 2014, 31(1):10-20. https://doi.org/10.1053/j. semdp.2014.01.006 PMID: 24680178

[5] Björnsson J, McLeod RA, Unni KK, Ilstrup DM, Pritchard DJ. Primary chondrosarcoma of long bones and limb girdles. Cancer, 1998, 83(10):2105-2119. PMID: 9827715

[6] Rosenthal DI, Schiller AL, Mankin HJ. Chondrosarcoma correlation of radiological and histological grade. Radiology, 1984, 150(1):21-26. https://doi.org/10.1148/radiology.150.1. 6689763 PMID: 6689763
[7] Ene R, Sinescu RD, Ene P, Popescu D, Cîrstoiu MM, Cîrstoiu FC. Proximal tibial osteosarcoma in young patients: early diagnosis, modular reconstruction. Rom J Morphol Embryol, 2015, 56(2): 413-417. PMID: 26193207

[8] Mercuri M, Picci P, Campanacci L, Rulli E. Dedifferentiated chondrosarcoma. Skeletal Radiol, 1995, 24(6):409-416. https:// doi.org/10.1007/BF00941235 PMID: 7481896

[9] Gelderblom H, Hogendoorn PCW, Dijkstra SD, van Rijswijk CS, Krol AD, Taminiau AHM, Bovée JVMG. The clinical approach towards chondrosarcoma. Oncologist, 2008, 13(3):320-329. https://doi.org/10.1634/theoncologist.2007-0237. Erratum in: Oncologist, 2008, 13(5):618. PMID: 18378543

[10] Leddy LR, Holmes RE. Chondrosarcoma of bone. Cancer Treat Res, 2014, 162:117-130. https://doi.org/10.1007/9783-319-07323-1 6 PMID: 25070233

[11] Dai X, Ma W, He X, Jha RK. Review of therapeutic strategies for osteosarcoma, chondrosarcoma, and Ewing's sarcoma. Med Sci Monit, 2011, 17(8):RA177-RA190. https://doi.org/10. 12659/msm.881893 PMID: 21804475 PMCID: PMC3539609

[12] Hanna SA, Whittingham-Jones $P$, Sewell MD, Pollock RC, Skinner JA, Saifuddin A, Flanagan A, Cannon SR, Briggs TWR. Outcome of intralesional curettage for low-grade chondrosarcoma of long bones. Eur J Surg Oncol, 2009, 35(12): 1343-1347. https://doi.org/10.1016/j.ejso.2009.06.001 PMID: 19570648

[13] Chen X, Yu LJ, Peng HM, Jiang C, Ye CH, Zhu SB, Qian WW. Is intralesional resection suitable for central grade 1 chondrosarcoma: a systematic review and updated meta-analysis. Eur J Surg Oncol, 2017, 43(9):1718-1726. https://doi.org/ 10.1016/j.ejso.2017.05.022 PMID: 28666625

[14] Italiano A, Mir O, Cioffi A, Palmerini E, Piperno-Neumann S, Perrin C, Chaigneau L, Penel N, Duffaud F, Kurtz JE, Collard O, Bertucci F, Bompas E, Le Cesne A, Maki RG, Ray Coquard I, Blay JY. Advanced chondrosarcomas: role of chemotherapy and survival. Ann Oncol, 2013, 24(11):29162922. https://doi.org/10.1093/annonc/mdt374 PMID: 24099780 PMCID: PMC3811906

[15] Moussavi-Harami F, Mollano A, Martin JA, Ayoob A, Domann FE, Gitelis S, Buckwalter JA. Intrinsic radiation resistance in human chondrosarcoma cells. Biochem Biophys Res Commun, 2006, 346(2):379-385. https://doi.org/10.1016/j.bbrc.2006.05.158 PMID: 16765318

[16] Fiorenza F, Abudu A, Grimer RJ, Carter SR, Tillman RM, Ayoub K, Mangham DC, Davies AM. Risk factors for survival and local control in chondrosarcoma of bone. J Bone Joint Surg $\mathrm{Br}$, 2002, 84(1):93-99. https://doi.org/10.1302/0301-6 20x.84b1.11942 PMID: 11837841

[17] Nota SPFT, Braun Y, Schwab JH, van Dijk CN, Bramer JAM. The identification of prognostic factors and survival statistics of conventional central chondrosarcoma. Sarcoma, 2015, 2015:623746. https://doi.org/10.1155/2015/623746 PMID: 26633939 PMCID: PMC4655064

[18] Cirstoiu C, Ene R, Panti Z, Ene P, Cirstoiu M. Particularities of shoulder recovery after arthroscopic Bankart repair with bioabsorbable and metallic suture anchors. Mater Plast, 2015, 52(3):361-363.

[19] Popescu D, Ene R, Popescu A, Cîrstoiu M, Sinescu R, Cîrstoiu C. Total hip joint replacement in young male patient with osteoporosis, secondary to hypogonadotropic hypogonadism. Acta Endocrinol (Bucharest), 2015, 11(1):109-113. https://doi.org/10.4183/aeb.2015.109

[20] Nica M, Cretu B, Ene D, Antoniac I, Gheorghita D, Ene R. Failure analysis of retrieved osteosynthesis implants. Materials (Basel), 2020, 13(5):1201. https://doi.org/10.3390/ma13051 201 PMID: 32155981 PMCID: PMC7085058

[21] Henderson ER, O'Connor MI, Ruggieri P, Windhager R, Funovics PT, Gibbons CL, Guo W, Hornicek FJ, Temple HT, Letson GD. Classification of failure of limb salvage after reconstructive surgery for bone tumours: a modified system including biological and expandable reconstructions. Bone Joint J, 2014, 96-B(11):1436-1440. https://doi.org/10.1302/ 0301-620X.96B11.34747 PMID: 25371453

[22] Mourikis A, Mankin HJ, Hornicek FJ, Raskin KA. Treatment of proximal humeral chondrosarcoma with resection and allograft. J Shoulder Elbow Surg, 2007, 16(5):519-524. https://doi.org/ 10.1016/j.jse.2006.10.010 PMID: 17521925

[23] Shen ZN, Nishida K, Doi H, Oohashi T, Hirohata S, Ozaki T, Yoshida A, Ninomiya Y, Inoue H. Suppression of chondro- 
sarcoma cells by 15 -deoxy-delta 12,14 -prostaglandin $\mathrm{J} 2$ is associated with altered expression of $\mathrm{Bax} / \mathrm{Bcl}-\mathrm{xL}$ and $\mathrm{p} 21$. Biochem Biophys Res Commun, 2005, 328(2):375-382. https:// doi.org/10.1016/j.bbrc.2004.12.186 PMID: 15694358

[24] Kim DW, Seo SW, Cho SK, Chang SS, Lee HW, Lee SE, Block JA, Hei TK, Lee FY. Targeting of cell survival genes using small interfering RNAs (siRNAs) enhances radiosensitivity of grade II chondrosarcoma cells. J Orthop Res, 2007, 25(6):820-828. https://doi.org/10.1002/jor.20377 PMID: 17343283

[25] Kim DW, Kim KO, Shin MJ, Ha JH, Seo SW, Yang J, Lee FY. siRNA-based targeting of antiapoptotic genes can reverse chemoresistance in P-glycoprotein expressing chondrosarcoma cells. Mol Cancer, 2009, 8:28. https://doi.org/10. 1186/1476-4598-8-28 PMID: 19445670 PMCID: PMC2689171

[26] Gitelis S, Bertoni F, Picci P, Campanacci M. Chondrosarcoma of bone. The experience at the Istituto Ortopedico Rizzoli. J Bone Joint Surg Am, 1981, 63(8):1248-1257. PMID: 7287795

[27] Leerapun T, Hugate RR, Inwards CY, Scully SP, Sim FH. Surgical management of conventional grade I chondrosarcoma of long bones. Clin Orthop Relat Res, 2007, 463:166-172. https://doi.org/10.1097/BLO.0b013e318146830f PMID: 17632422

[28] Bindiganavile S, Han I, Yun JY, Kim HS. Long-term outcome of chondrosarcoma: a single institutional experience. Cancer Res Treat, 2015, 47(4):897-903. https://doi.org/10.4143/crt. 2014.135 PMID: 25687868 PMCID: PMC4614192

[29] Teunis T, Nota SPFT, Hornicek FJ, Schwab JH, LozanoCalderón SA. Outcome after reconstruction of the proximal humerus for tumor resection: a systematic review. Clin Orthop Relat Res, 2014, 472(7):2245-2253. https://doi.org/10.1007/ s11999-014-3474-4 PMID: 24469551 PMCID: PMC4048415

[30] Streitbuerger A, Henrichs M, Gosheger G, Ahrens H, Nottrott M, Guder W, Dieckmann R, Hardes J. Improvement of the shoulder function after large segment resection of the proximal humerus with the use of an inverse tumour prosthesis. Int Orthop, 2015, 39(2):355-361. https://doi.org/10.1007/s00264 -014-2560-2 PMID: 25326856

[31] De Wilde L, Boileau P, Van der Bracht H. Does reverse shoulder arthroplasty for tumors of the proximal humerus reduce impairment? Clin Orthop Relat Res, 2011, 469(9): 2489-2495. https://doi.org/10.1007/s11999-010-1758-x PMID: 21210310 PMCID: PMC3148383

[32] Malawer M, Wittig J. Overview of resections around the shoulder girdle: anatomy, surgical considerations and classification. In: Malawer M, Sugarbaker PH (eds). Musculoskeletal cancer surgery: treatment of sarcomas and allied diseases. Springer, Dordrecht, 2004, 179-202. https://doi.org/10.1007/0-306-48 407-2_9

[33] Kamikawa M, Matsumura N, Okada K, Suzuki T, Nakayama R, Iwamoto T, Sato K, Nakamura M, Matsumoto M. Humeral head replacement with wrapping reconstruction of the rotator cuff after resection of chondrosarcoma with long-term shoulder function: a case report. J Med Cases, 2019, 10(2):53-57. https://doi.org/10.14740/jmc3252

[34] Albergo JI, Farfalli Luis GL, Ayerza MA, Muscolo DL, AponteTinao LA. Proximal humerus chondrosarcoma. Long-term clinical and oncological outcomes. Rev Esp Cir Ortop Traumatol, 2019, 63(3):181-186. https://doi.org/10.1016/j.recot.2019.01. 002 PMID: 30914237

[35] Mery B, Espenel S, Guy JB, Rancoule C, Vallard A, Aloy MT, Rodriguez-Lafrasse C, Magné N. Biological aspects of chondrosarcoma: leaps and hurdles. Crit Rev Oncol Hematol, 2018 , 126:32-36. https://doi.org/10.1016/j.critrevonc.2018.03.009 PMID: 29759564

\section{Corresponding author}

Mihai Nica, MD, Department of Orthopedics and Traumatology, Carol Davila University of Medicine and Pharmacy, 8 Eroii Sanitari Avenue, Sector 5, 050474 Bucharest, Romania; Phone +40762-300 209, e-mail: mikx99n@gmail.com

Received: May 26, 2020

Accepted: March 7, 2021 